\title{
Split Mouth Gingival Depigmentation with Scalpel and Diode Laser: A Comparative Study
}

\author{
Dr.Suneethi Margaret Dey ${ }^{1}$, Dr.Nagarathna D.V ${ }^{2}$, Dr.Catherine Jacob ${ }^{3}$ Dr.Jenny \\ Susan Roy \\ ${ }^{1,3,4}$ PG, Department of Periodontics, A J Shetty Institute of Dental Sciences, Mangalore, Karnataka, India. \\ ${ }^{2}$ Professor, Department of Periodontics, A J Shetty Institute of Dental Sciences, Mangalore, Karnataka, India.
}

\begin{abstract}
Melanin pigmentation often occurs in the gingiva as a result of excess deposition of melanin, due to which the gingiva appears black. Though, it is not pathological, many people complain of dark gums as unaesthetic. Gingiva is the most frequently pigmented intraoral tissue, with more pigmentation concentrated in the incisor region which may interfere with the smile line and patient may feel conscious about the appearance. In such patients, aesthetic periodontal plastic surgery can give excellent results. In this context we have attempted a case series of depigmentation in the anterior region. To compare the two techniques clinically, split mouth depigmentation procedure was carried out, using a semi-conductor diode laser and conventionally by scalpel. The laser treatment resulted in no post-operative bleeding and minimal post-operative pain as compared with scalpel technique, although there was no difference in effectiveness and aesthetic outcome.
\end{abstract}

Keywords: diode laser, melanin depigmentation, scalpel technique

\section{Introduction}

Smile plays an important role in improving esthetics and radiates health and self-confidence. One of the important aspects in a "smile" is the color of the gingiva. Several factors that determine the color are, number and size of blood vessels, thickness of the epithelium, extent of keratinization, and endogenous and exogenous pigmentation ${ }^{1}$. Oral pigmentation is a discoloration of the gingival/oral mucosa, and is associated with several exogenous and endogenous factors1. Melanin, carotene, reduced hemoglobin and oxyhemoglobin are the main pigments contributing to the normal color of the oral mucosa ${ }^{2}$.Etiological factors are varied, which include drugs, heavy metals, genetics, endocrine disturbances, syndromes such as Albright's syndrome, Peutz Jegher's syndrome, and also in inflammation. Adverse habits such as smoking can also stimulate melanin pigmentation, and the intensity of pigmentation is related to the duration of smoking and the number of cigarettes consumed. The pigmentation is mostly localized at the anterior labial gingiva, affecting females more than males ${ }^{3}$.The present case series describes the comparison between two surgical depigmentation technique The scalpel technique and the diode laser surgery.
1.1Techniques
Different techniques for depigmentation are ${ }^{3}$ :
1. Scalpel technique
2. Cryosurgery
3. Electrosurgery
4. Lasers
Nd: YAG(neodymium-doped yttrium aluminum garnet) laser,
Er: YAG (erbirum-doped yttrium aluminum garnet) laser,
Carbon dioxide $\left(\mathrm{CO}_{2}\right)$ laser
5. Chemical methods including acoustic agents - not used nowadays
a. $90 \%$ phenol and $95 \%$ alcohol
b. Ascorbic acid
6. Method aimed at masking the pigmented gingival from less pigmented gingival areas
a. Free gingival graft
b. Acellular dermal matrix allograft

\section{Case Report}

Five patients [four male, one female], aged 19-25 years who, visited the Department of Periodontology, A.J Institute of Dental Sciences, Mangalore with the chief complaint of "dark gums" were selected for this procedure. The patient's dental history revealed that the blackish discoloration of gingiva was present since birth, suggestive of physiologic melanin pigmentation. Clinical examination revealed pronounced 
bilateral melanin pigmentation associated with a healthy periodontium. Their medical history was noncontributory. The patients were in good general health and there were no contraindications for the surgeries. All depigmentation areas were graded from 1 to 2 according to the "Takeshi et al" scale of grading gingival depigmentation.

\subsection{Clinical evaluation}

Melanin pigmentation index (Takashi et al. ) $^{4}$

The degree of melanin pigmentation was determined by melanin pigmentation index with the scoring given as follows: [Figure A]

Score 0: No pigmentation

Score 1: Solitary unit(s) of pigmentation in papillary gingiva without extension between neighbouring solitary units

Score 2: Formation of continuous ribbon extending from neighbouring solitary units.

\subsection{Surgical Procedure}

The surgical procedure was performed under local anaesthesia. (Lignocaine with adrenaline in the ratio of 1:100,000 by weight). At the right maxillary anterior region from central incisor to canine diode laser of $810 \mathrm{~nm}$ with $3.5 \mathrm{~W}$ in continuous mode was used. Exposure parameters were set using the recommended guidelines, followed by careful removal of epithelium containing melanin layer (Figure C). There was absolutely no bleeding during and after the procedure.

On the left side, Bard-Parker handle with a \#15 blade was used for depigmentation (Figure D) Pressure was applied with a sterile gauze moistened in saline to control haemorrhage. High volume suction was used near the tissues. On completion of depigmentation, a periodontal dressing was placed over the surgical area and oral hygiene instructions were given to the patients. The patients were advised to use chlorhexidine mouthwash twice daily for 2 weeks postoperatively. Post-operatively the patients were prescribed, Non-steroidal antiinflammatory drugs, (Imol plus twice daily for three days). Patients were recalled after 1 week for re-evaluation. Wound healing was uneventful on both the sides (Figure E). Post-operative follow up after 1 month showed the areas were completely healed, with no signs of repigmentation (Figure F). Presently after 2 months, the patient is still on follow-up visits. Depigmentation was not carried out for mandibular anterior region because they were of no aesthetic concern for the patient.

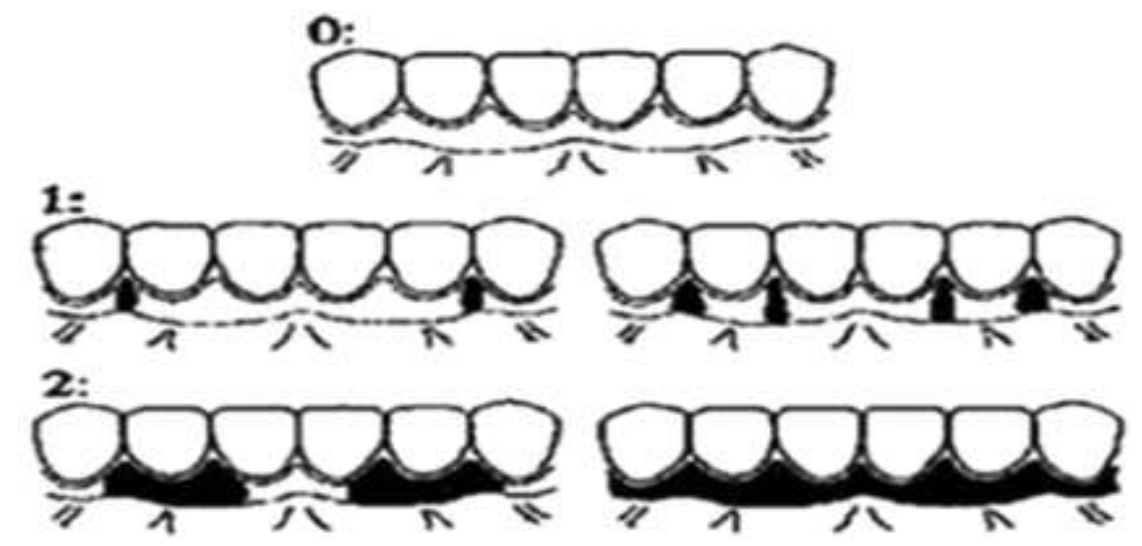

Fig. A Melanin pigmentation index (Takashi et al.)

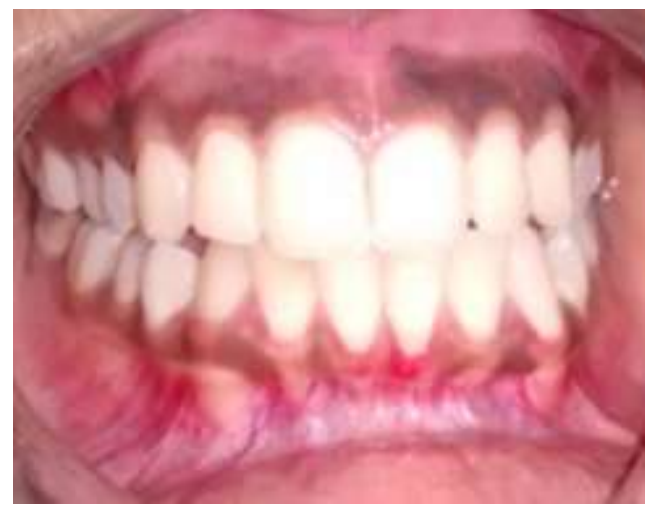

Fig. B Pre-operative

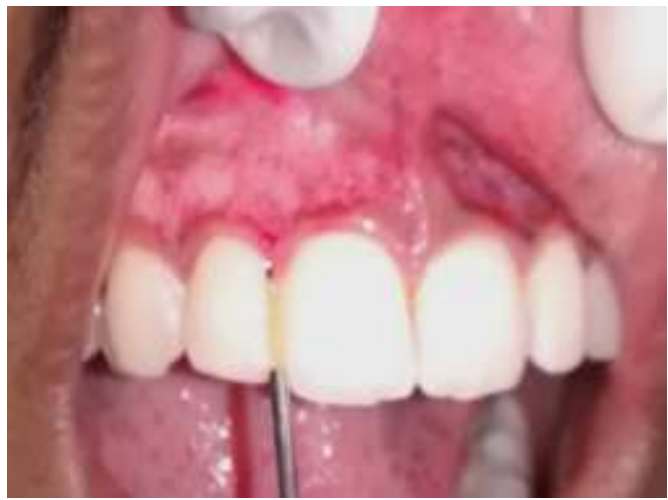

Fig. C LASER Technique 


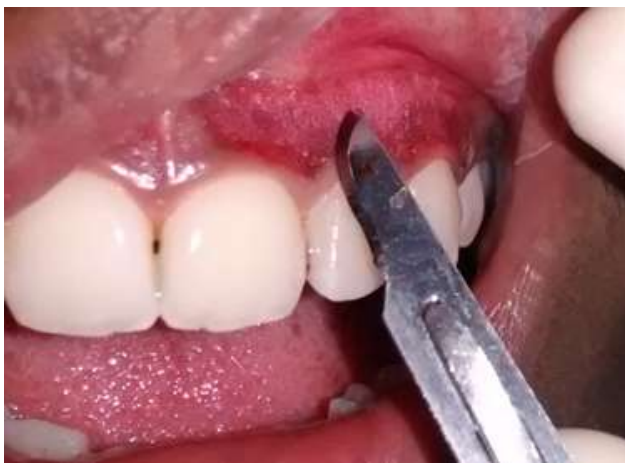

Fig. D Scalpel Technique

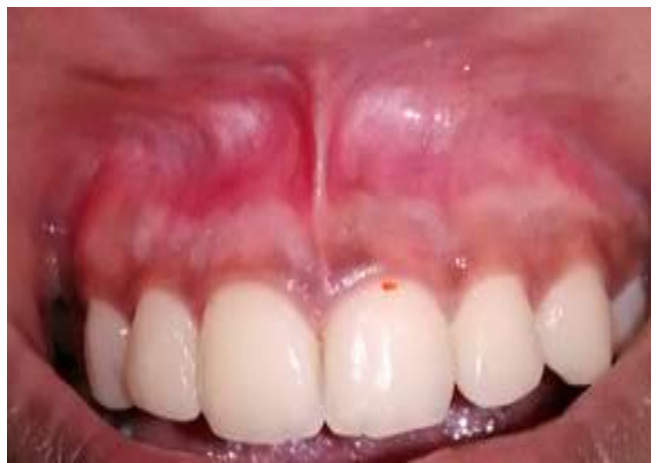

Fig. E 1 week post-operative

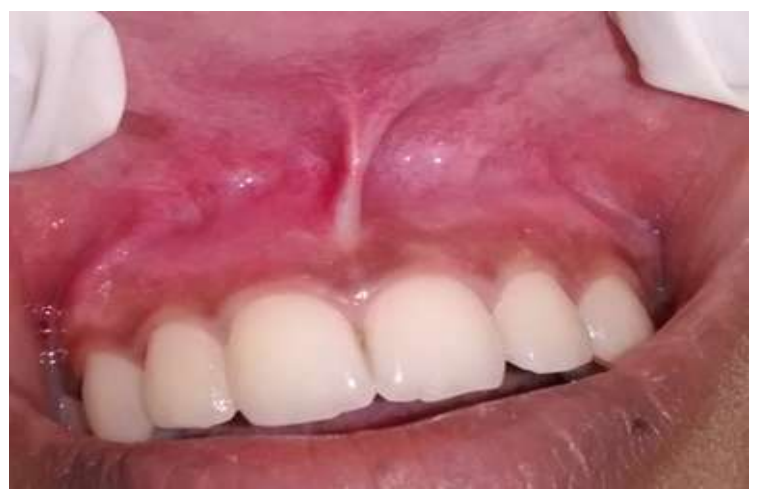

Fig. F 1 month post-operative

\section{Discussion}

Aesthetics has become the prime importance in this fast pace and self-conscious society and gingival hyperpigmentation is one of the most demanded periodontal aesthetic treatment. Gingival hyperpigmentation is caused by excessive deposition of melanin located in the basal and suprabasal cell layers of the epithelium. Melanin pigmentation is the result of melanin granules produced by melanoblasts intertwined between epithelial cells at the basal layer of the gingival epithelium. The degree of pigmentation varies from one person to another and depends on variety of factors especially the melanoblastic activity. Although melanin pigmentation of the gingiva is completely benign and does not present a medical problem, complaints of "black gums" are common, particularly in patients having a very high smile line (gummy smile) $)^{3}$.

Various treatment modalities have been carried out in the past for correction of pigmented gingiva with variable success ${ }^{6}$.One of the first, and still popular, techniques to be employed is the surgical removal of undesirable pigmentation using scalpels. The procedure essentially involves surgical removal of gingival epithelium along with a layer of the underlying connective tissue and allowing the denuded connective tissue to heal by secondary intention. The new epithelium that forms is devoid of melanin pigmentation ${ }^{7}$. With the advent of new technology in dentistry, lasers have taken over and have been used to ablate the cells containing and producing melanin pigment with appreciable success. Numerous laser systems such as the CO2, Nd: YAG, Er: YAG, and diode lasers have been successfully used for gingival depigmentation ${ }^{6}$.

\subsection{Advantages \& Disadvantages}

There are many advantages of LASER (Light Amplification by Stimulated Emission of Radiation) over surgical procedure, according to Wigdore et al (1995) they are ${ }^{4}$ :

- Dry and bloodless surgery

- Instant sterilization of surgical field

- $\quad$ Reduced bacteraemia

- $\quad$ Reduced mechanical trauma

- Minimal post-operative swelling and scarring

- Minimal post-operative pain

In this case, all the above-mentioned advantages were experienced. LASERS can be used in patients to reduce fear and anxiety. Also, less chair side time is required when comparing to the traditional techniques and hence results in more patient cooperation. There is increased coagulation and a necrotic slough is formed over the surface of the soft tissues ${ }^{4}$. 
However, LASER's have got few disadvantages and the following are:

- Expensive

- Technique sensitive

- Need of protective eye wear for doctor, patient as well as the assistant

\section{Conclusion}

In this current case series both scalpel and laser was found to be effective in the treatment of gingival hyperpigmentation. The use of diode laser appears to be a safe and effective alternate method, but further wellconducted randomized trials should be carried out to make the absolute conclusion.

\section{References}

[1]. Seda Ozturan, Aslihan Usumez. Case Report Aesthetic Treatment Of Gingival Hyperpigmentation by Er:YAG Laser. Journal of the Laser and Health Academy Vol. 2013, No.1.

[2]. Rahul Kathariya, A. R. Pradeep. Split mouth de-epithelization techniques for gingival depigmentation: A case series and review of literature. Journal of Indian Society of Periodontology - Vol 15, Issue 2, Apr-Jun 2011

[3]. Bhanu Murthy M, Jasjit Kaur, Rupali Das. Treatment of gingival hyperpigmentation with rotary abrasive, scalpel, and laser techniques: A case series. Journal of Indian Society of Periodontology - Vol 16, Issue 4, Oct-Dec 2012.

[4]. Takashi H, Tanaka K, Ojima M, Yuuki K. Association of melanin pigmentation in the gingiva of children with parents who smoke. Pediatrics 2005;116:e186-90

[5]. Amit Bhardwaj, Harpreet Singh Grover, Sanjay Lal. Gingival Depigmentation with Scalpel and Diode Laser. World Journal of Dentistry, October-December, 2012;3(4):359-362.

[6]. Kalakonda Butchibabu et al. Comparative evaluation of gingival depigmentation using a surgical blade and a diode laser. Journal of Dental Lasers- Vol 8, Issue 1, January - June 2014.

[7]. Kumara Ajeya E G, Avinash J L, Rajiv N P,Galgali Sushama R. Split Mouth Gingival Depigmentation Using Blade and Diode Laser- A Case Report. Annals of Dental Research (2011) Vol. 1 (1): 91-95. 